\title{
INSTANTANEOUS FREQUENCY ESTIMATION OF POLYNOMIAL PHASE SIGNALS USING LOCAL POLYNOMIAL WIGNER-VILLE DISTRIBUTION
}

\author{
Pu Wang ${ }^{1}$, Hongbin $\mathrm{Li}^{1}$, and Braham Himed ${ }^{2}$ \\ 1. Department of ECE, Stevens Institute of Technology, Hoboken, NJ 07030, USA \\ 2. AFRL/RYRT, 2241 Avionics Circle, Bldg 620, Dayton, OH 45433, USA
}

\begin{abstract}
This paper makes use of local polynomial Wigner-Ville distribution (LPWVD), originally designed for nonparametric instantaneous frequency (IF) estimation of transient signals, to propose a parametric IF estimation for polynomial phase signals (PPSs). Statistical performance such as asymptotic bias and variance of the LPWVD-based parametric IF estimator is derived in closed-form. Based on the analytical results, we extend the statistical efficiency of the Wigner-Ville distribution (WVD) for a second-order PPS only to that of the LPWVD for an arbitrary order, when the IF is estimated at the middle of sample observations. Simulation results verify the analytical performance and comparisons with the polynomial Wigner-Ville distribution (PWVD) show that the LPWVD-based parametric IF estimator can provide better performance.
\end{abstract}

Index Terms - Parametric method, instantaneous frequency, polynomial-phase signal.

\section{INTRODUCTION}

Instantaneous frequency (IF) of a signal is an important feature in many situations such as seismic, radar, sonar, communications, and biomedical applications $[1,2]$. Therefore, estimation of IF has received considerable attention over decades, see [2,3] and references therein.

Depending on assumptions on the signal model, an IF estimator can be considered as a nonparametric one or a parametric one [3]. Nonparametric IF estimators make no implicit assumptions about the form of the signal phase, and for this reason, exhibit high variance (see [3, Section III], [4] and references therein). In contrast, parametric IF estimators usually assume a prior on the signal phase, which can be expressed as a finite order polynomial, according to the Weierstrass theorem [3, Section IV]. By exploiting the a prior phase information, the parametric approach can produce an IF estimate with lower variance than the nonparametric one. In this paper, we emphasize the parametric approach to estimate the IF. For a $P$ thorder polynomial phase signal (PPS), the maximum likelihood estimation (MLE) has to search over a $P$-dimensional space for the IF estimate [3]. To relief the computational burden, a number of suboptimal parametric methods are proposed [3,5-7]. Among those, the high-order ambiguity function (HAF) and the polynomial WignerVille distribution (PWVD) are the most popular choices for a PPS with an arbitrary order [6,7]. Statistical performance of the above two parametric IF estimators has been well studied in [6] and [8], respectively.

This work was supported in part by the Air Force Research Laboratory (AFRL) under Contract FA8750-05-2-0001 and the Air Force Office of Scientific Research (AFOSR) under Grant FA9550-09-1-0310
In this paper, we make use of the local polynomial Wigner-Ville distribution (LPWVD), originally devised for the nonparametric IF estimation $[4,9]$, to propose a parametric estimator for the IF estimation of a PPS. It is shown that the well-known Wigner-Ville distribution (WVD) for a linear FM signal (a second-order PPS) [5] can be interpreted as a special case of the parametric LPWVD for a PPS with an arbitrary order. Different from the highly nonlinear HAF- and PWVD-based parametric IF estimators, the LPWVDbased parametric estimator utilizes a bilinear transformation only, which in turns yields smaller MSEs at high SNR and a lower SNR threshold. The statistical performance of the LPWVD-based parametric IF estimator is derived in closed-forms via a first-order perturbation analysis. The results suggest that, the LPWVD-based parametric IF estimator is asymptotically efficient at high SNR when the IF is estimated at the middle point of observations, which is a desired property has not been claimed by either the HAF-based estimator or the PWVD-based one.

\section{SIGNAL MODEL AND PARAMETRIC IF ESTIMATION}

A $P$ th-order PPS is defined

$$
s(n)=A \exp \{j \phi(n)\}=A \exp \left\{j \sum_{i=0}^{P} a_{i} n^{i}\right\},
$$

where $A$ is the constant amplitude, $\phi(n)$ is the instantaneous phase (IP) and $\left\{a_{i}\right\}_{i=0}^{P}$ are unknown phase parameters, respectively. The IF $\Omega(n)$ is defined as the first derivative of the IP [1], i.e.,

$$
\Omega(n)=\frac{d \phi(n)}{d n}=\sum_{i=1}^{P} i a_{i} n^{i-1}
$$

As indicated in [8, Section VI], a properly designed PWVDbased approach can outperform the HAF-based approach when the IF is estimated at the middle point of observations. As such, we focus on the PWVD-based parametric IF estimation as a reference to the proposed LPWVD-based approach. For the above PPS, the PWVD is defined as

$$
\operatorname{PWVD}_{s}(n, \omega)=\sum_{m=-M}^{M} \prod_{q=1}^{Q / 2} s\left(n+d_{q} m\right) s^{*}\left(n-d_{q} m\right) e^{-j \omega m}
$$

where $\omega$ denotes index in the IF domain, $2 M+1$ is the length of a rectangular window, $Q$ is the order of the PWVD, and $d_{q}$ is the lagcoefficient of the PWVD. To ensure the PWVD is centered around the IF, the PWVD order $Q$ and the coefficient set $d_{q}$ should satisfy

\section{U.S. Government work not protected by U.S. copyright}


a number of constraints; see (5) and (6) of [7]. When $Q=2$, the PWVD reduces to the well known WVD [5] as

$$
\operatorname{WVD}_{s}(n, \omega)=\sum_{m=-M}^{M} s(n+m) s^{*}(n-m) e^{-j \omega m} .
$$

It is seen that the PWVD is a higher-order extension of the WVD by updating a bilinear transformation in (4) to successive $Q / 2$ bilinear transformations in (3), while retaining the computational efficiency of the WVD, i.e., one-dimensional (1-D) searching in the $\omega$ axis only.

The LPWVD $[4,9]$, on the other hand, extends the WVD in a manner of replacing the one-dimensional matched filter by a multidimensional matched filter, while keeping the second-order nonlinearity of the WVD which is essential to the performance at low SNR. The LPWVD for a PPS in (1) is defined as

$$
W_{s}(n, \boldsymbol{\omega})=\sum_{m=-M}^{M} s(n+m) s^{*}(n-m) e^{-j \sum_{l=1}^{L} \frac{2 \omega_{l}}{(2 l-1) !} m^{2 l-1}},
$$

where $\boldsymbol{\omega}=\left[\omega_{1}, \omega_{2}, \cdots, \omega_{L}\right]^{T}$ denotes indices in $L$ odd-order phase-derivatives, and $L=\lceil P / 2\rceil$. Specifically, $\omega_{1}$ is the IF index. Replacing $s(n)$ with the PPS in (2), it can be verified that the LPWVD is maximized at $\boldsymbol{\omega}_{0}=\left[\Omega(n), \phi^{(3)}(n), \cdots, \phi^{(2 L-1)}(n)\right]^{T}$, where $\phi^{(l)}(n)$ denotes the $l$ th phase derivative. In other words, the $L$ odd-order phase derivatives of the PPS can be estimated from the maximum of the LPWVD. It is also noted that, when $L=1$, the LPWVD reduces to the WVD in (4).

It is noted that the LPWVD-based nonparametric IF estimator for a PPS in white Gaussian noise is analyzed in [4]. The results show that the the LPWVD-based nonparametric approach yields a biased IF estimate, and the bias is proportional to the window length, while the variance of the estimate error is inversely proportional to the window length. A carefully designed scheme has been proposed to reduce the MSE of this nonparametric IF estimator [4]. In the following, however, we show that the LPWVD-based parametric IF estimator is unbiased and, the MSE of the LPWVD-based parametric IF estimator reaches the CRB at high SNR, when the IF is estimated at the middle point of observations.

\section{STATISTICAL PERFORMANCE OF THE LPWVD-BASED PARAMETRIC IF ESTIMATOR}

Consider a noisy PPS $x(n)=s(n)+v(n)$, where $v(n)$ is a complex white Gaussian noise with zero mean and variance $\sigma^{2}$. The LPWVD for the noisy PPS can be defined similarly as (5):

$$
W_{x}(n, \boldsymbol{\omega})=\sum_{m=-M}^{M} x(n+m) x^{*}(n-m) e^{-j \sum_{l=1}^{L} \frac{2 \omega_{l}}{(2 l-1) !} m^{2 l-1}} .
$$

Note that the LPWVD is real, i.e., $W_{x}(n, \boldsymbol{\omega})=W_{x}^{*}(n, \boldsymbol{\omega})$. As a result, for a given time, the LPWVD-based parametric IF estimator can be expressed as

$$
\left[\hat{\Omega}(n), \hat{\phi}^{(3)}(n), \cdots, \hat{\phi}^{(2 L-1)}(n)\right]^{T}=\arg \max _{\boldsymbol{\omega}} W_{x}(n, \boldsymbol{\omega}),
$$

where the first estimate $\hat{\Omega}(n)$ gives the IF estimate.
Due to the noise, the resulting LPWVD can be separated into a signal component $W_{s}(n, \boldsymbol{\omega})$ and an interference component $W_{v s}(n, \boldsymbol{\omega})$ as

$$
W_{x}(n, \boldsymbol{\omega})=W_{s}(n, \boldsymbol{\omega})+W_{v s}(n, \boldsymbol{\omega}),
$$

where $W_{s}(n, \boldsymbol{\omega})$ is given by (5) and $W_{v s}(n, \boldsymbol{\omega})$ is given by

$$
W_{v s}(n, \boldsymbol{\omega})=\sum_{m=-M}^{M} C_{v s}(n, m) e^{-j \sum_{l=1}^{L} \frac{2 \omega_{l}}{(2 l-1) !} m^{2 l-1}},
$$

with

$$
\begin{aligned}
C_{v s}(n, m)= & s(n+m) v^{*}(n-m)+v(n+m) s^{*}(n-m) \\
& +v(n+m) v^{*}(n-m) .
\end{aligned}
$$

The interference component, acts like a perturbation, moves the IF estimate from $\boldsymbol{\omega}_{0}$ to $\boldsymbol{\omega}_{0}+\delta \boldsymbol{\omega}$, where $\delta \boldsymbol{\omega}$ is the estimate error. In the following, a quantitative analysis for the estimate error $\delta \boldsymbol{\omega}$ is obtained in closed-forms via a multivariate first-order perturbation analysis, which is summarized without any proof.

Proposition 1: For a $P$ th-order noisy PPS defined above, the $L$ phase-derivative estimates obtained in (7) are all asymptotically unbiased and their asymptotic MSEs are given by:

$$
E\left\{\left(\delta \omega_{l}\right)^{2}\right\}=\frac{\left(1+\frac{1}{2 \mathrm{SNR}}\right)}{4 M^{4 l-1} \mathrm{SNR}} \frac{[(2 l-1) !]^{2} \prod_{i=1}^{L}(2 l+2 i-1)^{2}}{(4 l-1) \prod_{\substack{i=1 \\ i \neq l}}^{L}(2 l-2 i)^{2}},
$$

where $l=1,2, \cdots, L$, and the SNR is defined as $A^{2} / \sigma^{2}$. Specifically, the MSE of the LPWVD-based parametric IF estimate is

$$
E\left\{\left(\delta \omega_{1}\right)^{2}\right\}=\frac{3\left(1+\frac{1}{2 \mathrm{SNR}}\right)}{4 M^{3} \mathrm{SNR}} \prod_{i=2}^{L} \frac{(2 i+1)^{2}}{(2 i-2)^{2}} .
$$

From the above Proposition 1, the MSEs of the $L$ phasederivative estimates are independent of the phase parameters $\left\{a_{i}\right\}_{i=0}^{P}$ of the PPS. At high SNR, the MSEs are all approximately proportional to $\mathrm{SNR}^{-1}$, while at low SNR they are proportional to $\mathrm{SNR}^{-2}$. Moreover, the $l$ th estimate, i.e., $\omega_{l}$, has an MSE inversely proportional to $M^{4 l-1}$. Hence, the larger the window length, the lower the MSE. As such, for a given SNR and time $n$, the asymptotic MSEs of the estimates are minimized by using the maximum window length given by 1) $M=\frac{N-1}{2}-\left|\frac{N-1}{2}-n\right|$ in the asymmetric sampling case with $n \in\{0, \cdots, N-1\}$; and 2) $M=\frac{N-1}{2}-|n|$ in the symmetric sampling case with $n \in\left\{-\frac{N-1}{2}, \cdots, \frac{N-1}{2}\right\}$, where $N$ is the number of samples and we assume $N$ is odd.

\subsection{Case of $L=1$ : Convergence to the WVD}

It is meaningful to look at the LPWVD with $L=1$ which reduces to the discrete WVD [5] as well as the second-order PWVD [8]: According to (11) with $l=1$ and $L=1$, the variance of the estimate error reduces to

$$
E\left\{(\delta \Omega)^{2}\right\}=\frac{3\left(1+\frac{1}{2 \mathrm{SNR}}\right)}{4 M^{3} \mathrm{SNR}}
$$

which agrees with the results in [8] (see the equation above (27) of [8]) and (7) of [5], when the IF is estimated at the at the middle of observations (i.e., replace $M$ with $N / 2$ in (13)). 


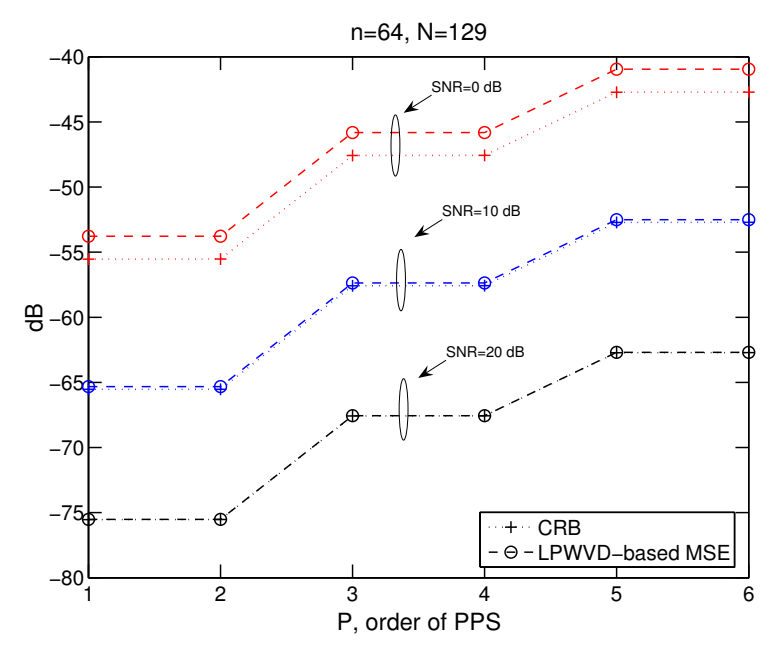

Fig. 1. The MSE of the LPWVD-based parametric IF estimate and CRB versus the order of the PPS

\subsection{Comparison to the CRB}

In [10], the CRB for parametric IF estimation was established for a general PPS model, i.e., the phase of the PPS can be expressed as a linear combination of Legendre polynomials. The explicit CRB expressions for the PPS in (1) with order 1 through 3 have also been derived. According to Proposition 1, the MSE of the LPWVD-based parametric IF estimate is maximized at the middle point of observations for a given SNR, $P$, and $N$ :

$$
E\left\{\left(\delta \omega_{1}\right)^{2}\right\}=\frac{6\left(1+\frac{1}{2 \mathrm{SNR}}\right)}{N^{3} \mathrm{SNR}} \prod_{i=2}^{L} \frac{(2 i+1)^{2}}{(2 i-2)^{2}}
$$

A numerical comparison between the above MSE and corresponding CRB of [10] is shown in Fig. 1 when $n=64$ and $N=129$. The results verify that, at high SNR, the performance of the LPWVDbased parametric IF estimator reaches the CRB at the middle point of observations for the PPS with order 1 to 6 . In other words, the LPWVD-based parametric estimator is asymptotically efficient at high SNR when the IF is estimated at the middle point. At low SNR, the MSE of the LPWVD-based parametric IF estimate exhibits a performance loss with respect to the $\mathrm{CRB}$ due to $\mathrm{SNR}^{-2}$. For example, at $\mathrm{SNR}=0 \mathrm{~dB}$, the performance loss is about $3 \mathrm{~dB}$.

\section{SIMULATION RESULTS}

In this section, the performance of the PWVD-based and LPWVDbased parametric IF estimators will be evaluated. The simulated MSEs of the LPWVD-based parametric IF estimate allow us to confirm our theoretical results in Section 3. All simulated results are based on 500 Monte-Carlo simulations.

\subsection{A Third-Order PPS}

A third-order PPS is generated with parameters $A=1,\left\{a_{0}, a_{1}, a_{2}, a_{3}\right\}=$ $\left\{0,0.1 \pi,-\pi /(3 N), \pi /\left(6 N^{2}\right)\right\}$, and $N=129$. In this case, [7] proposed a sixth-order PPS with coefficients $\left\{d_{1}, d_{2}, d_{3}\right\}=$ $\{0.62,0.75,0.87\}$. Its MSE for the IF estimate is approximated by

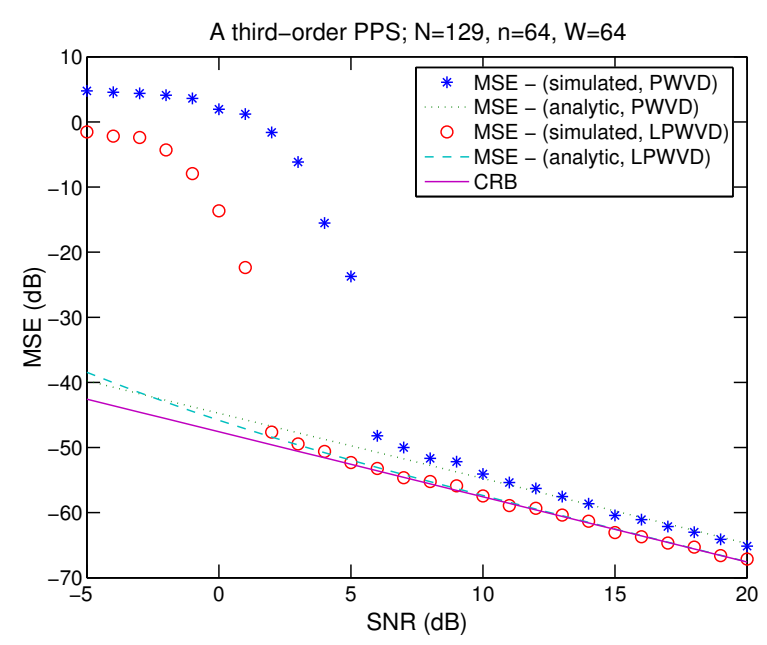

Fig. 2. MSEs of the PWVD-based and the LPWVD-based parametric IF estimate versus the SNR for a third-order PPS, when $n=64$ and $N=129$

ignoring the second-order or higher order noise terms [8]:

$$
E\left\{(\delta \omega)^{2}\right\}_{\mathrm{PWVD}} \approx \frac{9}{M^{3} \mathrm{SNR}}
$$

where $M$ is the window size used in the PWVD. On the other hand, we need to use the LPWVD with $L=2$ :

$$
W_{x}\left(n, \omega_{1}, \omega_{2}\right)=\sum_{m} x(n+m) x^{*}(n-m) e^{-j\left(2 \omega_{1} m+\frac{\omega_{2}}{3} m^{3}\right)} .
$$

According to Proposition 1, the resulting MSE for the LPWVDbased IF estimate is

$$
E\left\{(\delta \omega)^{2}\right\}=\frac{75\left(1+\frac{1}{2 \mathrm{SNR}}\right)}{16 M^{3} \mathrm{SNR}} .
$$

Fig. 1 shows the MSE versus the SNR when the IF is estimated at the middle of observations, i.e., $n=64$. From this plot, it is clear that the LPWVD-based IF estimate provides smaller MSE than the PWVD-based estimate at SNR above $5 \mathrm{~dB}$. Compared with the PWVD-based estimate, the SNR threshold of the LPWVD-based one is about 4-dB lower. Moreover, the theoretical MSEs obtained in Proposition 1 shows a good prediction on the true performance of the LPWVD-based IF estimate. Again, the simulated MSEs verify that the LPWVD-based estimate approaches to the CRB at high SNR.

The MSE versus time point is also evaluated when SNR = $20 \mathrm{~dB}$ and shown in Fig. 3. At each time point $n$, the window size is $M=\min \{n, N-1-n\}$. From this figure, it is shown that the MSE and CRB are seen to be symmetric with respect to the middle point $n=64$. The numerical results are seen to agree with the theoretical results. Again, the LPWVD-based IF estimator shows lower MSEs than those of the sixth-order PWVD-based estimator.

\subsection{A Fifth-order PPS}

A higher-order PPS is also considered in this section. For a fifthorder PPS, the PWVD used in simulation is the eighth-order one in [7] with coefficients $\left\{d_{1}, d_{2}, d_{3}, d_{4}\right\}=\{0.316,0.316,0.316,-0.45\}$, 


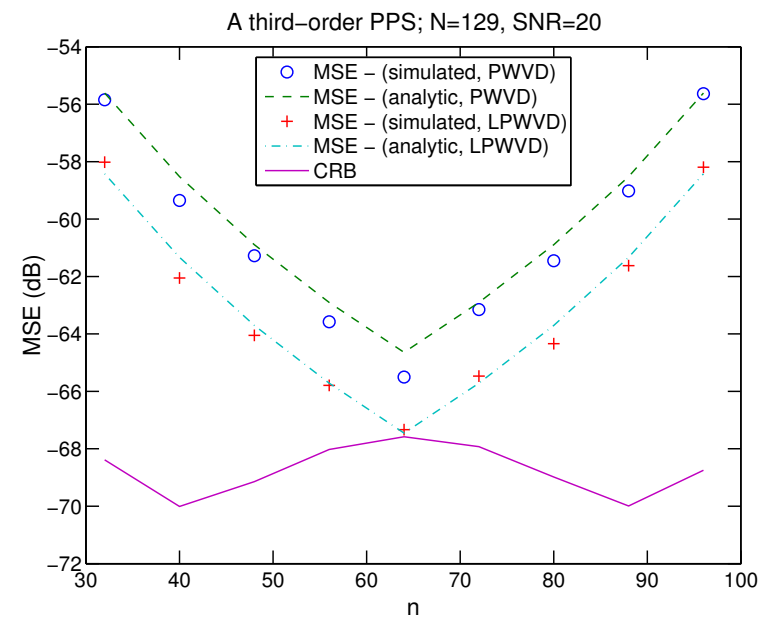

Fig. 3. MSEs of the PWVD-based and the LPWVD-based parametric IF estimate versus time for a third-order PPS, when SNR = $20 \mathrm{~dB}$ and $N=129$

while the LPWVD with $L=3$ is used

$$
\begin{aligned}
W_{x}\left(n, \omega_{1}, \omega_{2}, \omega_{3}\right)= & \sum_{m} x(n+m) x^{*}(n-m) \\
& \times e^{-j\left(2 \omega_{1} m+\frac{\omega_{2} m^{3}}{3}+\frac{\omega_{3} m^{5}}{60}\right)} .
\end{aligned}
$$

Corresponding theoretical expressions of the MSE are

$$
\begin{aligned}
E\left\{(\delta \omega)^{2}\right\}_{\mathrm{PWVD}} & \approx \frac{240}{N^{3} \mathrm{SNR}}, \\
E\left\{(\delta \omega)^{2}\right\} & =\frac{3675\left(1+\frac{1}{2 \mathrm{SNR}}\right)}{32 M^{3} \mathrm{SNR}},
\end{aligned}
$$

when the IF is estimated at the middle of observations, i.e., $M=$ $N / 2$. The MSE versus the SNR at $n=64$ is shown in Fig. 4 for a fifth-order PPS. It can be seen that the LPWVD-based IF estimate maintains its performance with respect to the CRB and retains its SNR threshold. In contrary, the MSE of the PWVD-based estimate increases due to the higher-order nonlinear transformation, and the SNR threshold also moves to about $12 \mathrm{~dB}$ for a fifth-order PPS from about $6 \mathrm{~dB}$ for a third-order PPS. This figure also confirms that our theoretical results agree with the simulated results when the SNR is above its threshold.

\section{CONCLUSION}

This paper proposed the local polynomial Wigner-Ville distribution as a parametric IF estimator for a PPS. Our systematic review shows that the LPWVD-based parametric IF estimator is in between the MLE and the PWVD-based parametric estimator for a tradeoff between the statistical performance and the computational complexity. Closed form analytical performance has been obtained via a firstorder perturbation principle. The results show that the LPWVDbased parametric estimator is unbiased and approaches to the CRB at high SNR from an asymptotic sense. Future study will emphasize on using both multilinear and multidimensional extension of the WVD for statistical and computational more efficiency of the IF estimate.

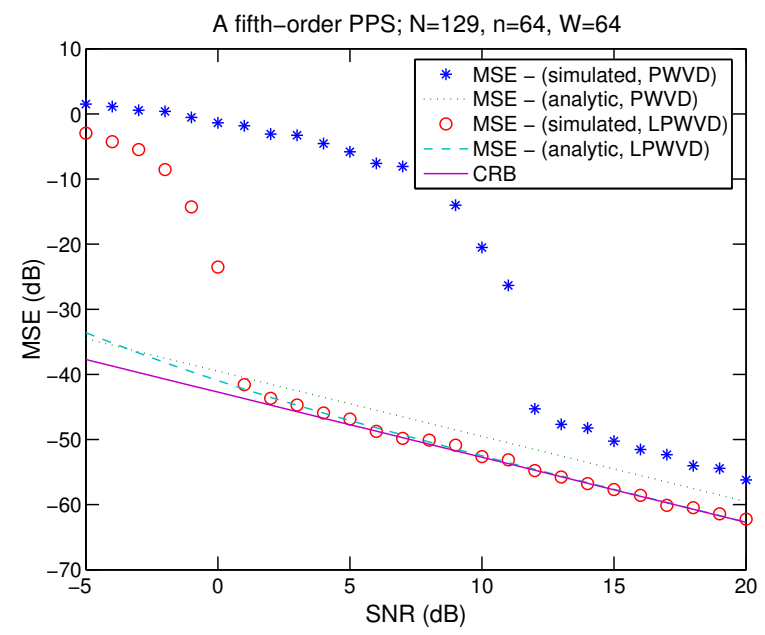

Fig. 4. MSEs of the PWVD-based and the LPWVD-based parametric IF estimate versus time for a fifth-order PPS, when $n=64$ and $N=129$

\section{REFERENCES}

[1] B. Boashash, "Interpreting and estimating the instantaneous frequency of a signal, Part 1: Fundamentals," Proc. IEEE, vol. 80, no. 4, pp. 520-538, April 1992.

[2] L. Cohen, Time-Frequency Analysis, Englewood Cliffs, Prentice-Hall, NJ, 1995.

[3] B. Boashash, "Interpreting and estimating the instantaneous frequency of a signal, Part 2: Algorithms and Applications," Proc. IEEE, vol. 80, no. 4, pp. 540-568, April 1992.

[4] V. Katkovnik, "Nonparametric estimation of instantaneous frequency," IEEE Trans. on Info. Theory, vol. 43, no. 1, pp. 183189, January 1997.

[5] P. Rao and F. J. Taylor, "Estimation of the instantaneous frequency using the discrete Wigner distribution," Electronics Letters, vol. 26, no. 4, pp. 246-248, Feburary 1990.

[6] B. Porat and B. Friedlander, "Asymptotic statistical analysis of the high-order ambiguity function for parameter estimation of polynomial-phase signals," IEEE Trans. on Info. Theory, vol. 42, no. 3, pp. 995-1001, May 1996.

[7] B. Barkat, "Design of higher order polynomial Wigner-Ville distributions," IEEE Transactions on Signal Processing, vol. 47, no. 9, pp. 2608-2611, September 1999.

[8] B. Barkat and B. Boashash, "Instantaneous frequency estimation of polynomial FM signals using the peak of the PWVD: Statistical performance in the presence of additive Gaussian noise," IEEE Trans. on Signal Processing, vol. 47, no. 9, pp. 2480-2490, September 1999.

[9] L. Stanković, "Local polynomial Wigner distribution," Signal Processing, vol. 59, pp. 123-128, 1997.

[10] S. Peleg, B. Porat, and B. Friedlander, "The achievable accuracy in estimating the instantaneous phase and frequency of a constant amplitude signal," IEEE Trans. on Signal Processing, vol. 41, no. 6, pp. 2216-2224, June 1993. 